NASA Contractor Report 178104

ICASE REPORT NO. $86-26$

NASA-CR-178104

19860017076
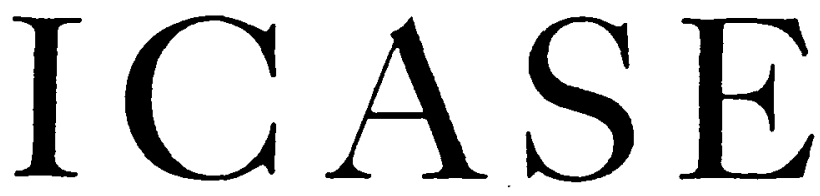

ON SIMILARITY SOLUTIONS OF A BOUNDARY LAYER

PROBLEM WITH AN UPSTREAM MOVING WALL

M. Y. Hussaini

W. D. Lakin

A. Nachman

Contract Nos. NAS1-17070, NAS1-18107

May 1986

INSTITLTE FOR CONPUTER APPLICATIONS IN SCIENCE AND ENGINEERING

NASA Langley Research Center, Hampton, Virginia 23665

Operated by the Universities Space Research Association

\section{NMSA}

National Aeronautics and

Space Administration

Longley Research Center

Hampton. Virginia 23665

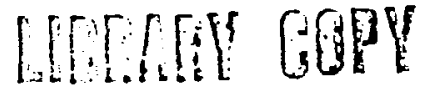

a) $1: 1980$

LANGLEY FESEARCH CEIVTER

LIBRARY, NASA

HAMMTON, VIRGINIA 


\title{
ON SIMILARITY SOLUTIONS OF A BOUNDARY LAYER PROBLEM \\ WITH AN UPSTREAM MOVING WALL
}

\author{
M. Y. Hussaini \\ Institute for Computer Applications in Science and Engineering \\ W. D. Lakin \\ old Dominion Univeristy \\ and \\ Institute for Computer Applications in Science and Englneering \\ A. Nachman \\ Air Force Office of Scientific Research \\ Dedicated to Milton E. Rose \\ on Occasion of his 60 th Birthday
}

\begin{abstract}
This work deals with the problem of a boundary layer on a flat plate which has a constant velocity opposite in direction to that of the uniform mainstream. It has previously been shown that the solution of this boundary value problem is crucially dependent on the parameter which is the ratio of the velocity of the plate to the velocity of the free stream. In particular, it was proved that a solution exists only if this parameter does not exceed a certain critical value, and numerical evidence was adduced to show that this solution is nonunique. Using Crocco formulation the present work proves this nonuniqueness. Also considered are the analyticity of solutions and the derivation of upper bounds on the critical value of wall veloctty parameter.
\end{abstract}

Research for the first and second authors was supported by the National Aeronautics and Space Administration under NASA Contract Nos. NAS1-17070 and NAS1-18107 while they were in residence at ICASE, NASA Langley Research Center, Hampton, VA 23665-5225. 



\section{Introduction}

The boundary layer on the upstream-moving flat plate at zero incidence admits of the classical similarity transformation which reduces the relevant partial differential equations to the Blasius equation.

$$
\begin{aligned}
f^{\prime \prime}+f f^{\prime \prime} & =0 \\
f(0) & =0 \\
f^{-}(0) & =-\lambda, \quad \lambda>0 \\
f^{-}(\infty) & =1,
\end{aligned}
$$

where $f=\psi(x, y) / \sqrt{(2 v x)}, \psi$ being the dimensional stream function, and $\nu$ the kinematic viscosity, and $\eta=y / \sqrt{(2 v x)}$. This equation can be readily integrated once to yield

i.e.,

$$
f^{\prime \prime}(n)=f^{\prime \prime}(0) \exp \left[-\int_{0}^{n} f(z) d z\right]
$$

$$
f^{\prime \prime}(\eta)=f^{\prime \prime}(0) \exp \left[\frac{1}{2} \lambda \eta^{2}-\frac{1}{2} \int_{0}^{n}(\eta-z)^{2} f^{\prime \prime}(z) d z\right]
$$

using integration by parts twice. Obviously, the shear stress $f^{\prime \prime}(n)$ has the same sign as the skin-friction at the wall, $f^{\prime \prime}(0)$. For $\lambda=0$, Weyl proved the existence and uniqueness using function-theoretical methods. For $\lambda \leq 0$, Callegari and Friedman and Callegari and Nachman found it expedient to work with the Crocco formulation, that is, in terms of shear stress $g\left(=f^{\prime \prime}\right)$ as the dependent variable and tangential velocity $u\left(=f^{-}\right)$as the independent variable: 


$$
\begin{aligned}
g(u) g^{\prime \prime}(u)+u & =0,-\lambda<u<1, \\
g^{-}(-\lambda) & =0 \\
g(1) & =0 .
\end{aligned}
$$

For $\lambda \leq 0$, they proved existence, uniqueness, and analyticity of solutions to Eq. (2) using an analytical function theory approach. For the case $\lambda>0$, Hussaini and Lakin proved that a solution exists only for $\lambda$ less than a critical value $\lambda_{c}$. Their numerical results showed nonuniqueness for $\lambda \leq \lambda_{c}$, and the numerical value of $\lambda_{c}$ was found to be $0.3541 \ldots$. In this work, the nonuniqueness is established rigorously. Also, proof of analyticity, and absolute monotonicity etc., is given. Certain analytical upper bounds on $\lambda$ are established.

For convenience, we use the transformation $\mathrm{x}=\mathrm{u}+\lambda$ to map the interval $-\lambda<u<1$, to $0<x<1+\lambda$. So we consider the equations

$$
\begin{gathered}
g(x) g^{\prime \prime}(x)+(x-\lambda)=0, \quad 0<x<1+\lambda \\
g^{-}(0)=0 \\
g(1+\lambda)=0 .
\end{gathered}
$$

\section{Analyticity of Solutions}

In this section, the following basic result will be proved:

THEOREM 1: There is a range of positive values of $\lambda$ such that the positive continuous solution $g(x)$ of the boundary value problem (1.1) and (1.2) is analytic on the closed interve1 $[0,1+\lambda]$. 
This theorem will be proved by considering a sequence of lemmas. The first lemma required is:

LEMMA 1: The derivative $g^{-}(x)$ vanishes at one and only one point on the interval $0<x<1+\lambda$. Further, $g(x)$ has its maximum value at this point.

Proof of Lemma 1: Equation (1.1) can be integrated using the initial condition $g^{\prime}(0)=0$ to give

$$
g^{-}(x)=\int_{0}^{x} \frac{\lambda-\xi}{g(\xi)} d \xi,
$$

Thus, as the initial value $\alpha=g(0)>0$, both $g(x)$ and $g^{-}(x)$ are positive for $0<x \leq \lambda$. Also,

$$
g^{\prime \prime}(x)=(\lambda-x) / g(x)
$$

is positive for $0 \leq x<\lambda$ and $g^{\prime \prime}(\lambda)=0$. The continuous solution $g(x)$ remains positive for $\lambda<x<1+\lambda$, and hence $g^{\prime \prime}(x)$ is now negative. This gives that $g^{-}(x)$ is a monotone decreasing function for $x>\lambda$. As

$g^{-}(1+\lambda)=-\infty$, there must thus be at least one point on the interval

$(\lambda, 1+\lambda)$ at which $g^{-}(x)$ vanishes. In fact, assuming that $g^{-}(x)$ vanishes at more than one point leads to a contradiction, for suppose that $g^{-}$vanishes at both $\mathrm{x}_{1}$ and $\mathrm{x}_{2}$ with $\mathrm{x}_{1}<\mathrm{x}_{2}$. Then, $\mathrm{g}^{\prime \prime}$ would have to vanish at least once between these two points which is impossible as $g^{\prime \prime}\langle 0$ for $x>\lambda$. The proof of Lemma 1 is concluded by noting that $g^{\prime \prime}\left(x_{1}\right)<0$ implies that $g\left(x_{1}\right)$ must be the maximum value of $g(x)$. 
LEMMA 2: The solution $g(x)$ has a convergent power series expansion on the closed interval $\left[\mathrm{x}_{1}, 1+\lambda\right]$.

Proof of Lemma 2: As $g(x)$ is positive and differentiable for

$\mathrm{x}_{1} \leq \mathrm{x}<1+\lambda$, equation (2.2) shows that $\mathrm{g}(\mathrm{x})$ has derivatives of all orders on this interval. Further, expressions for these derivatives may be obtained directly from the differential equation (1.1). Induction shows that for $n \geq 1$, derivatives of $g(x)$ satisfy the recursion relation

$$
g^{(n+3)}=-\frac{1}{g}\left\{(n+1) g^{-} g^{(n+2)}+\frac{1}{2} \sum_{k=2}^{n+1}\left[\left(\begin{array}{c}
n+1 \\
n-k+3
\end{array}\right)+\left(\begin{array}{c}
n+1 \\
k
\end{array}\right) g^{(k)}\right] g^{(n-k+3)}\right\}
$$

where $g(k)$ is the $k$-th derivative of $g$ with respect to $x$ and $\left(\begin{array}{l}p \\ q\end{array}\right)$ is the usual combinatorial symbol.

Let $g\left(x_{1}\right)=\beta$, and consider the auxilliary function $G(x)$ defined by

$$
G(x)=B-g(x)
$$

Then, as $B$ is the maximum value of $g(x), G(x)$ is non-negative for $x_{1}<x<1+\lambda$. Also, for all $n \geq 1, G^{n}(x)=-g^{(n)}(x)$. Consequently, equation (2.1) shows that $G^{-}(x)$ is positive on the interval $x \leq x<1+\lambda$. From $(1.1)$,

$$
G^{\prime \prime}(x)=\frac{x-\lambda}{g(x)} \text { and } G^{-}(x)=\frac{1+G^{-} G^{\prime \prime}}{g(x)}
$$

are also both positive on this interval. The recursion relation (2.3) thus shows that all derivatives of $G(x)$ are non-negative on the closed interval $\left[\mathrm{x}_{1}, 1+\lambda-\varepsilon\right]$ where $1+\lambda-\mathrm{x}_{1}>\varepsilon>0$. Hence, $\mathrm{G}(\mathrm{x})$ is absolutely 
monotonic on this closed interval. A theorem of Bernstein [4] now gives that $G(x)$ has a convergent Taylor series expansion about the point $x_{1}$ whose radius of convergence is not less than $1+\lambda-x_{1}$. From the definition of $G(x)$, it immediately follows that for $\left|x-x_{1}\right|<1+\lambda-x_{1}, g(x)$ has the convergent expansion

$$
g(x)=\sum_{n=0}^{\infty} \frac{g^{(n)}\left(x_{1}\right)}{n !}\left(x-x_{1}\right)^{n}
$$

Application of a Tauberian theorem [5] further shows that the power series (2.5) converges at the singular point $x=1+\lambda$ to the value $g(1+\lambda)=0$ completing the proof of Lemma 2 .

To establish Theorem 1, it must be shown that for a nontrivial range of positive values of $\lambda$, the power series (2.5) for the solution $g(x)$ of the boundary value problem (1.1) and (1.2) converges at the left boundary point $\mathrm{x}=0$. This will be accomplished in Lemma 3. A consequence of this convergence will be an expansion for the initial value of $g(x)$ as the series

$$
\alpha=\beta+\sum_{n=2}^{\infty} \frac{(-1)^{n} x_{1}^{n}}{n !} g^{(n)}\left(x_{1}\right) .
$$

LEMMA 3: There exists a positive value $\bar{\lambda}$ such that if $0<\lambda<\bar{\lambda}$ then $x_{1}<(1+\lambda) / 2$.

Lemma 3 gives that the left-hand boundary point $x=0$ lies inside the radius of convergence of the power series expansion (2.5). Consequently, the corresponding solution of the boundary value problem will be analytic. It should be noted that the upper bound on $x_{1}$ given in Lemma 3 is a sufficient, but not a necessary, condition for convergence. 
Proof of Lemma 3: Equation (1.1) may be integrated from 0 to $x$ using the identity $g^{\prime \prime}=\left(g g^{-}\right)^{-}-\left(g^{-}\right)^{2}$ and the initial condition $g^{-}(0)=0$. A second integration from 0 to $x_{1}$ now gives the result

$$
\frac{x_{1}^{2}\left(x_{1}-3 \lambda\right)}{6}=\frac{\alpha^{2}-\beta^{2}}{2}+\int_{0}^{x_{1}}\left(x_{1}-\xi\right) g^{-2}(\xi) d \xi .
$$

An upper bound on the right-hand side of (2.7) and a lower bound on the maximum point $x_{1}$ are now required to establish the lemma.

A lower bound on $x_{1}$ may be obtained by using (2.1) and the fact that $g^{-}\left(x_{1}\right)=0$ to obtain

$$
\int_{0}^{\lambda} \frac{\lambda-\xi}{g(\xi)} d \xi=\int_{\lambda}^{x_{1}} \frac{\xi-\lambda}{g(\xi)} d \xi
$$

As $g(x)$ is monotone increasing on $\left[0, x_{1}\right], g(x) \leq g(\lambda)$ on $[0, \lambda]$, but $g(\lambda) \leq g(x)$ on $\left[\lambda, x_{1}\right]$. Equation (2.8) now gives

$$
x_{1} \geq 2 \lambda
$$

As $g(x)$ has its only maximum at $x_{1}$ by Lemma 1 , an immediate lower bound on $g\left(x_{1}\right)=\beta$ is $\beta>\alpha$. A sharper lower bound on $\beta$ can be obtained from the expression

$$
\beta=\alpha+\int_{0}^{x_{1}} \frac{\left(x_{1}-\xi\right)(\lambda-\xi)}{g(\xi)} d \xi=\alpha+\int_{0}^{x_{1}} \frac{(\lambda-\xi)^{2}}{g(\xi)} d \xi
$$

obtained by integrating (2.1) from 0 to $x_{1}$. As $g(x) \leq \beta$, and by (2.9), $x_{1}-\lambda \geq \lambda$, equation (2.10) now gives the quadratic inequality 


$$
\beta^{2}-\alpha \beta-\frac{2 \lambda^{3}}{3} \geq 0
$$

which implies

$$
B \geq \frac{\alpha+\sqrt{\alpha^{2}+8 \lambda^{3 / 3}}}{2} \text {. }
$$

A lower bound on $\beta^{2}-\alpha^{2}$ which follows from $(2.12)$ is thus

$$
\beta^{2}-\alpha^{2} \geq \frac{2 \lambda^{3}}{3}
$$

Consider next bounds on the initial value $\alpha$. Let $x=1+\lambda$. Then, integrating (2.1) from 0 to $X$ and using $g(X)=0$ gives

$$
\alpha=\int_{0}^{X} \frac{(x-\xi)(\xi-\lambda)}{g(\xi)} d \xi
$$

This relation may be rewritten in terms of strictly positive integrals as

$$
\alpha=\int_{\lambda}^{X} \frac{(x-\xi)(\xi-\lambda)}{g(\xi)} d \xi-\int_{0}^{\lambda} \frac{(x-\xi)(\lambda-\xi)}{g^{-}(\xi)} d \xi
$$

which shows

$$
\alpha \leq \int_{\lambda}^{X} \frac{(x-\xi)(\xi-\lambda)}{g(\xi)} d \xi
$$

The convexity of $g(x)$ on $[\lambda, x]$ implies that on this interval

$g(x) \geq g(\lambda) \cdot(X-x)$. Equation (2.16) now gives that $\alpha \leq(2 g(\lambda))^{-1}$. As

$\alpha<g(\lambda)$, this further implies

$$
\alpha^{2} \leq 1 / 2
$$


Equation (2.15) does not lend itself to the derivation of a lower bound on $\alpha^{2}$. However, in the present consideration of analyticity, the required bound can be obtained from a relation between $\alpha$ and $B$ which follows from the existence proof of Hussaini and Lakin [3]. This proof shows that if $\lambda$ is positive and does not exceed a critical value, there is at least one initial value $\alpha$ such that a positive continuous solution of the initial value problem consisting of $(1.1)$ and the conditions $g(0)=\alpha$ and $g^{-}(0)=0$ exists and has $g(X)=0$, i.e., it is a solution of the boundary value problem. Further, the solution of the initial value problem will be unique if

$$
\beta<2 \alpha \text {. }
$$

It must be noted that a unique solution of the initial value problem dnos not imply a unique solution of the boundary value problem. This will be shown in section 4 .

A lower bound on $\alpha^{2}$ follows by using (2.18) in (2.12). The result is

$$
\alpha^{2} \geq \frac{\lambda^{3}}{3}
$$

The final bound needed for use in equation (2.7) is an upper bound for $g^{-}(x)$ on the interval $\left[0, x_{1}\right]$. From $(2.2), g^{\prime \prime}(x)$ is a monotone decreasing function on this interval. Further, $g^{\prime \prime}(\lambda)=0$ while the third derivative of $g$ is negative when $x=\lambda$. Thus, $g^{-}(x)$ has its maximum value at $x=\lambda$. This implies that on $\left[0, x_{1}\right]$

$$
0 \leq g^{-}(x) \leq g^{-}(\lambda)=\int_{0}^{\lambda} \frac{\lambda-\xi}{g(\xi)} d \xi .
$$


As $g(x) \geq \alpha$ on $[0, \lambda]$, equation (2.20) gives

$$
0 \leq g^{-}(x) \leq \frac{\lambda^{2}}{2 \alpha}
$$

An upper bound on the integral in equation (2.7) is thus

$$
\int_{0}^{x_{1}}\left(x_{1}-\xi\right) g^{-2}(\xi) d \xi \leq \frac{3}{8} \lambda x_{1}^{2}
$$

Use of (2.13) and (2.22) in equation (2.7) implies

$$
x_{1}^{2}\left(x_{1}-\frac{21}{4} \lambda\right)+2 \lambda^{3} \leq 0
$$

This relation gives that $x_{1}$ will be less than $x / 2$ for $\lambda$ in the range $0<\lambda<\bar{\lambda}=0.1176$. The sufficient condition for analyticity is thus satisfied for a range of positive values of $\lambda$ establishing Lemma 3 and Theorem 1 .

Equation (2.9) implies that $x_{1}$ cannot be less than $x / 2$ if $\lambda>1 / 3$. Indeed, direct numerical solution of the boundary value problem shows that $\mathrm{x}_{1}$ $<\mathrm{X} / 2$ when $\lambda<\hat{\lambda}=0.32$ and $\alpha$ lies on the upper branch in Figure 1 . The gap between the values of $\bar{\lambda}$ and $\hat{\lambda}$ is associated with fundemental problems in obtaining sharper bounds on the initial value $\alpha$. For example, equation (2.15) implies

$$
\alpha \leq \int_{\lambda}^{x_{1}} \frac{(x-\xi)(\xi-\lambda)}{g(\xi)} d \xi+\int_{x_{1}}^{X} \frac{(\xi-\lambda)}{g(\xi)} d \xi-\int_{x_{1}}^{X} \frac{(\xi-\lambda)^{2}}{g(\xi)} d \xi .
$$


Individually, the last two integrals in (2.24) are formally infinite, yet they must cancel so as to give an order one upper bound. Direct numerical calculations show that the upper bound on $\alpha^{2}$ is $\alpha^{2}<0.219961$. The upper bound in (2.17) is thus conservative by over a factor of two.

It must again be noted that $x_{1}<x / 2$ is only a sufficient condition for analyticity. For values of $\alpha$ on the upper branch of Figure 1, solutions of the boundary value problem can thus be expected to remain analytic for $\lambda$ greater than $\hat{\lambda}$. Further insight can be gained by examining parameter values for which the condition (2.18), which is sufficient for a unique solution of the associated initial value problem, is maintained. Numerical results show that (2.18) holds for all values of $a$ on the upper branch of Figure 1 . It also holds for $\alpha$ on the lower branch of Figure 1 in the relatively small range $0.351<\lambda<\lambda_{c}$ and is violated over the remainder of the lower branch. The behavior of $\beta$ as a function of $\alpha$ is given in Figure 2. For values of $\lambda$ associated with initial values on much of the lower branch of Figure 1, there must thus be serious doubts as to whether solutions of the boundary value problem (1.1) and (1.2) are analytic.

\section{An Opper Bound on $\lambda_{c}$}

The existence proof of Hussaini and Lakin [3] established the existence of solutions of $(1.1)$ and $(1.2)$ for positive values of $\lambda$ less than a critical value $\lambda_{c}$. It was shown from (1.1) and (1.2) that $\lambda_{c}<1 / 2$. The value of $\lambda_{c}$ was also determined numerically in that work to be

$$
\lambda_{c}=0.3541079 \ldots
$$


In this section, additional upper bounds for $\lambda_{c}$ will be obtained directly from $(1.1)$ and $(1.2)$.

Using the identity that precedes equation (2.7), equation (2.1) can be integrated from 0 to $x$ and the result integrated again from 0 to $x$. As $g(X)=0$, this gives

$$
\frac{x^{2}(x-3 \lambda)}{6}=\frac{\alpha^{2}}{2}+\int_{0}^{X}(x-\xi) g^{-2}(\xi) d \xi
$$

The right-hand side of (3.2) is intrinsically positive, and thus

$$
x-3 \lambda \geq 0
$$

This relation immediately implies

$$
\lambda \leq 1 / 2
$$

To obtain sharper bounds now requires the use of positive lower bounds for $\alpha^{2}$ and the integral in (3.2). While no additional assumptions are required to obtain (3.4), in what follows it will be necessary to assume that $\beta<2 \alpha$. However, as noted previously, this condition is satisfied on the entire upper branch in Figure 1 . In particular, it is satisfied in the limiting case when $\lambda=\lambda_{c}$.

Let the integral $I(x)$ be defined by

$$
I(x)=\int_{0}^{x}(x-\xi) g^{-2}(\xi) d \xi
$$

Then, as $I(X)>0$, equation (3.2) implies 


$$
x^{2}(x-3 \lambda) \geq 3 \alpha^{2}
$$

Replacing $X$ by $1+\lambda$ and using (2.19) now gives the inequality

$$
3 \lambda^{3}+3 \lambda^{2}-1<0
$$

which yields the improved bound

$$
\lambda<0.47533 .
$$

A slightly sharper bound can be obtained by noting that $I(X)>I(\lambda)$. Let $\delta=g(\lambda)$. Then, $g(x) \leq \delta$ on $[0, \lambda]$, so on this interva1

$$
g^{-2}(x) \geq \frac{x^{2}}{4 \delta^{2}}(2 \lambda-x)^{2}
$$

This leads to the relation

$$
I(\lambda) \geq \frac{\lambda^{5}}{120 \delta^{2}}(5 \lambda+16)
$$

An upper bound on $\delta$ now follows from the fact that $g(x) \geq \alpha$ on $[0, \lambda]$ and

$$
\delta=\alpha+\int_{0}^{\lambda} \frac{(\xi-\lambda)^{2}}{g(\xi)} d \xi
$$

In particular,

$$
\delta^{2} \leq \frac{2 \lambda^{3}+1}{2}
$$

Use of (3.12) in (3.10) then shows 


$$
I(\lambda) \geq \frac{\lambda^{5}(5 \lambda+16)}{60\left(2 \lambda^{3}+1\right)}
$$

Equation (3.2) now gives

$$
x^{2}(X-3 \lambda) \geq 3 \alpha^{2}+6 I(\lambda)
$$

which leads to the inequality

$$
65 \lambda^{6}+76 \lambda^{5}+10 \lambda^{3}+30 \lambda^{2}-10 \leq 0 .
$$

The solution of $(3.15)$ is

$$
\lambda<0.46824
$$

which is only a marginal improvement over (3.8).

Even if the lower bound on $I(X)$ is further sharpened by considering this integral on the full interval $[0, \mathrm{X}]$, a significant decrease in the bound on $\lambda$ is not obtained. Again, this is due to the difficulties associated with obtaining sufficiently sharp bounds on the initial value $\alpha$.

4. Non-uniqueness of Solutions for $0<\lambda<\lambda_{c}$

Using direct numerical results, Hussaini and Lakin [3] have shown that if $\lambda$ is positive and less than $\lambda_{c}$ then solutions of the boundary value problem are not unique. For a fixed value of $\lambda$ in this range, as shown in Figure 1 there are two initial values $\alpha$ which lead to solutions of the boundary value problem. The purpose of this section is to prove this non- 
uniqueness directly from (1.1) and (1.2). To this end, it is convenient to consider the normalized initial value problem

$$
\begin{gathered}
h h^{\prime \prime}+t-L=0, \\
h(0)=1, \quad h^{-}(0)=0
\end{gathered}
$$

obtained from the initial value problem for $g(x)$ by taking

$$
g(x)=\alpha h(t) \text { with } x=\alpha^{2 / 3} t
$$

The parameter $L$ in $(4.1)$ is related to $\alpha$ and $\lambda$ through the expression

$$
L=\alpha^{-2 / 3} \lambda
$$

If $h(T)=0$ and $\alpha(\lambda)$ is given by

$$
\alpha=\{(1+\lambda) / T\}^{3 / 2}
$$

then $g(X)=0$, so the solution of the initial value problem with initial value (4.5) will also be a desired solution of the boundary value problem. Equations (4.3) through (4.5) also imply that in terms of $T$ and $I$

$$
\lambda=\frac{\mathrm{L}}{\mathrm{T}-\mathrm{L}}
$$

and

$$
\alpha=(T-L)^{-3 / 2} \text {. }
$$


LEMMA 4: Let $h_{1}(t)$ and $h_{2}(t)$ be solutions of the initial value problem (4.1) and (4.2) corresponding to $\mathrm{L}$ values $\mathrm{L}_{1}$ and $\mathrm{L}_{2}$, respectively. Then, if $L_{2}>L_{1}, h_{2}(t)>h_{1}(t)$.

Proof of Lemma 4: For $t \ll L, h(t)$ must be of the form $1+L t^{2} / 2$. Thus, the lemma holds for small values of $t$. That it holds for $0<t \leq T$ can now be shown by contradiction. Let $\bar{t}$ be the first value of $t$ at which $h_{1}(\bar{t})=h_{2}(\bar{t})$. As $h_{1}$ was previously less than $h_{2}$, this requires $h_{2}^{\prime \prime}(\bar{t})<h_{1}^{\prime \prime}(\bar{t})$. But,

$$
h_{2}^{\prime \prime}(\bar{t})=\frac{L_{2}-\bar{t}}{h_{2}(\bar{t})}=\frac{L_{2}-\bar{t}}{h_{1}(\bar{t})}>\frac{L_{1}-\bar{t}}{h_{1}(\bar{t})}=h_{1}^{\prime \prime}(\bar{t}) .
$$

This contradiction establishes Lemma 4 . Lemma 4 also shows that if $h_{1}\left(T_{1}\right)=0$ and $h_{2}\left(T_{2}\right)=0$, then $h_{2}\left(T_{1}\right)>0$. This implies that:

COROLLARY: $\mathrm{T}_{2}>\mathrm{T}_{1}$.

The derivative $h^{-}(t)$ is given by an expression analogous to equation (2.1). As $h(0)$ is positive, both $h(t)$ and $h^{-}(t)$ will be positive for $0 .<t \leq \mathrm{L}$. This shows that $\mathrm{T}>\mathrm{L}$. Consequently, the denominators in $(4.6)$ are strictly positive. The following lemma gives a sharper result:

LEMMA 5: $\mathrm{T}>3 \mathrm{~L}$.

Proof of Lemma 5: Equation (4.1) may be integrated twice from 0 to $t$ using (4.2) to give 


$$
1 / 2 h^{2}(t)+\frac{t^{3}}{6}-\frac{L t^{2}}{2}=1 / 2+\int_{0}^{t}(t-\xi) h^{-2}(\xi) d \xi
$$

This implies

$$
h^{2}(t)+\frac{1}{3} t^{2}(t-3 L) \geq 0
$$

Setting $t=T$ and $h(T)=0$ now establishes the lemma.

Consider next the behavior of $T$ as a function of $L$. It has already been shown in Lemma 4 that $T$ is a monotone increasing function of $L$.

LEMMA 6: $T(L)$ is superlinear in $L$.

Proof of Lemma 6: Let $t_{1}$ be the point at which $h^{-}\left(t_{1}\right)=0$. As is the case for the original initial value problem in the variable $x$, there is one and only one such point, it lies in the interval $I<t<T$, and $h\left(t_{I}\right)$ is a maximum value.

Equation (4.1) may be multiplied by $h^{-}$and divided by $h$ to give

$$
h h^{\prime \prime}+\frac{h^{-}(t-L)}{h}=0 \text {. }
$$

Integration from 0 to $t$ produces the result

$$
1 / 2 h^{-2}+(t-L) \operatorname{lnh}(t)-\int_{0}^{t} \operatorname{lnh}(\xi) \mathrm{d} \xi=0
$$

Evaluating $(4.12)$ at $t_{1}$ now shows 


$$
t_{1}=L+\frac{\int_{0}^{t_{1}} \operatorname{lnh}(\xi) d \xi}{\operatorname{lnh}\left(t_{1}\right)} .
$$

Next, the expression

$$
h(t)=1+\int_{0}^{t} \frac{(t-\xi)(L-\xi)}{h(\xi)} d \xi
$$

may be evaluated at $t=L$ to give an expression for $h(L)$.

$$
h(L)=1+\int_{0}^{L} \frac{(L-\xi)^{2}}{h(\xi)} d \xi .
$$

As $h^{-}(t)$ is non-negative on the interval $[0, L], h(t)$ is monotone increasing, so $h(t) \leq h(L)$. Use of this fact in (4.15) gives the quadratic inequality

$$
h^{2}(L)-h(L)-\frac{L^{3}}{3} \geq 0
$$

which implies $h^{2}(L) \geq L^{3} / 3$. The solution $h(t)$ has its maximum value at $t_{1}$. Consequently,

$$
h\left(t_{1}\right)>\sqrt{\frac{L^{3}}{3}} .
$$

One additional bound is needed before demonstrating the superlinear behavior of $T(L)$. The change of concavity of $h(t)$ on the interval $\left[0, t_{1}\right]$ due to the fact that $h^{\prime \prime}(L)=0$ precludes obtaining as a lower bound for $h$ on this interval the straight line which passes through the origin and the point $\left(t_{1}, h\left(t_{1}\right)\right)$, i.e., it cannot be shown that $h(t)>h\left(t_{1}\right) \cdot t / t_{1}$. However, for a given $L$, it is clear that $h(t)$ can be bounded below on this interval by a curve of the form 


$$
H(t ; k)=\frac{h\left(t_{1}\right) t^{k}}{t_{1}^{k}}
$$

for a value of $k>1$. As $k$ increases, these curves become progressively more convex. It should be noted that if $H(t, \bar{k})$ provides a lower bound on $\left[0, t_{1}\right]$ for the solution of $(4.1)$ and $(4.2)$ associated with $L=\bar{L}$, then, by Lemma $4, H(\tau, \bar{k})$ also provides a lower bound for solutions associated with larger values of $L$.

This lower bound for $h(t)$ on $\left[0, t_{1}\right]$ may be used to obtain an lower bound for the integral in equation (4.13). In particular,

$$
\int_{0}^{t} \operatorname{lnh}(\xi) d \xi>t_{1} \operatorname{lnh}\left(t_{1}\right)-k t_{1} .
$$

Equation (4.13) now implies that

$$
t_{1}>\frac{L}{k} \operatorname{lnh}\left(t_{1}\right)
$$

Use of (4.17) then gives

$$
T>t_{1}>\frac{L}{2 k} \ln \left(\frac{L}{3^{1 / 3}}\right)
$$

The superlinear behavior of $T(L)$ is thus established.

THEOREM 2: For positive values of $\lambda$ in the range $0<\lambda<\lambda_{c}$, solutions of the boundary value problem (1.1) and (1.2) are not unique. 
$-19-$

Proof of Therm 2: Consider the behavior of $L$ as a function of $\lambda$. By (4.4), $L(0)=0$. Equation (4.6) and the superlinear behavior of $T$ with repent to $\mathrm{L}$ shown in Lemma 6 now imply that the graph of $\lambda$ vs $\mathrm{L}$ must be as in Figure 3. In particular, for a fixed positive $\lambda$ which is less than $\lambda_{c}$, there will be two distinct values of L. By the corollary to Lemma 4 , each value of $\mathrm{L}$ must correspond to a different value of $T$. Equation (4.5) now shows that for the fixed value of $\lambda$, two distinct values $\alpha_{1}$ and $\alpha_{2}$ exist such that the solutions of the initial value problems with these $\alpha^{-} s$ are solutions of the boundary value problem (1.1) and (1.2). Solutions of the boundary value problem are thus not unique completing the proof of Theorem 2 . 


\section{References}

[1] A. J. Callegari and M. B. Friedman, "An analytical solution of a nonlinear, singular boundary value problem in the theory of viscous flows," J. Math. Anal. Appl., 21 (1968), pp. 510-529.

[2] A. J. Callegari and A. Nachman, "Some singular, nonlinear differential equations arising in boundary layer theory," J. Math. Anal. Appl., 64 (1978), pp. 96-105.

[3] M. Y. Hussaini and W. D. Lakin, "Existence and nonuniqueness of simflarity solutions of a boundary-layer problem," Quart. J. Mech. Appl. Math., 39 (1986), in press.

[4] A. F. Tinman, Theory of Approximations of Functions of a Real Variable, Pergamon Press, England, 1963.

[5] N. Wiener, The Fourier Integral and Certain of its Applications, Dover, New York, 1933. 


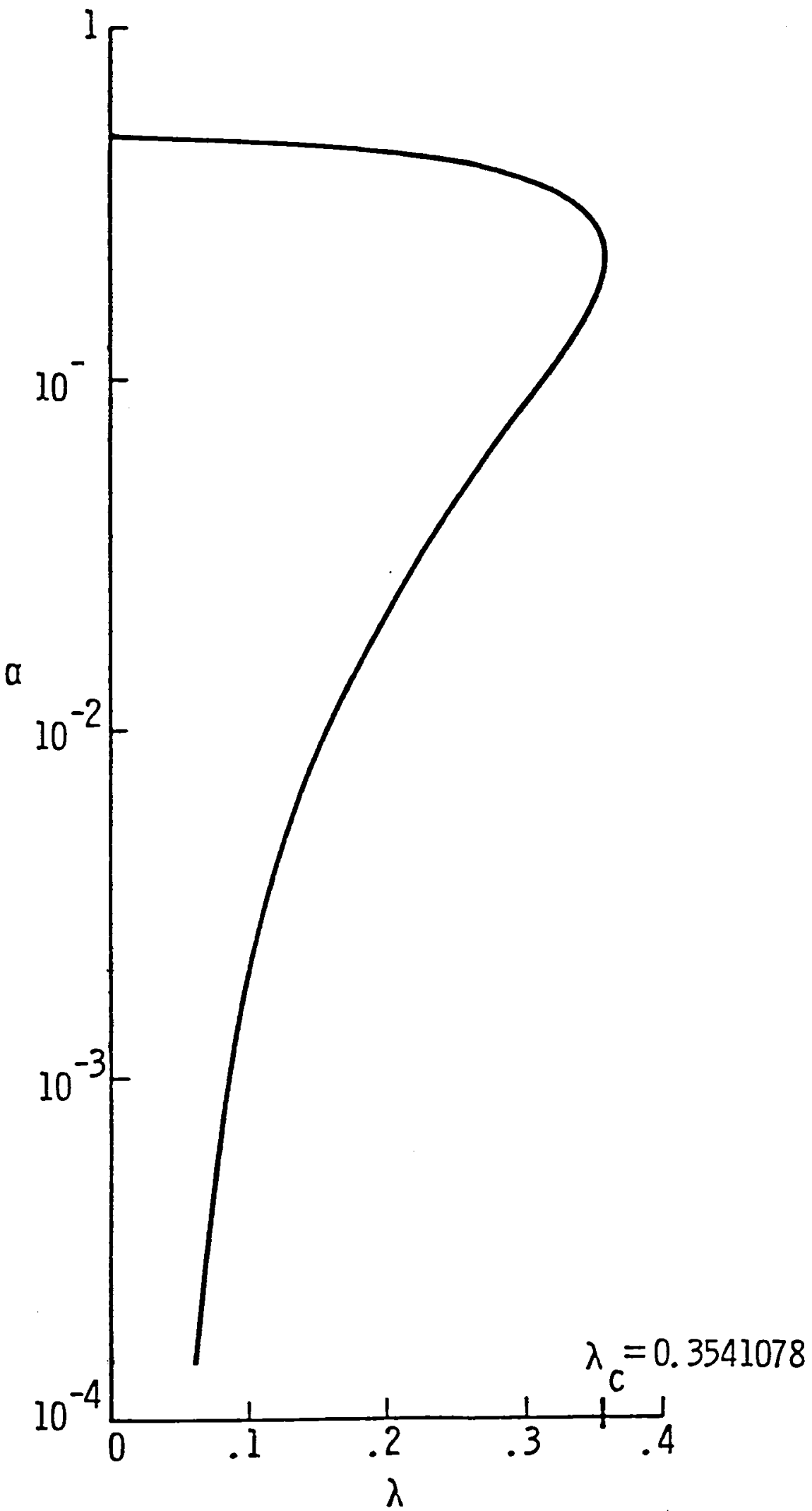

Figure 1. Values of the parameter $\alpha=f^{\prime \prime}(0)$ for which $f^{-}(\infty)=1$ as a function of $\lambda$. 


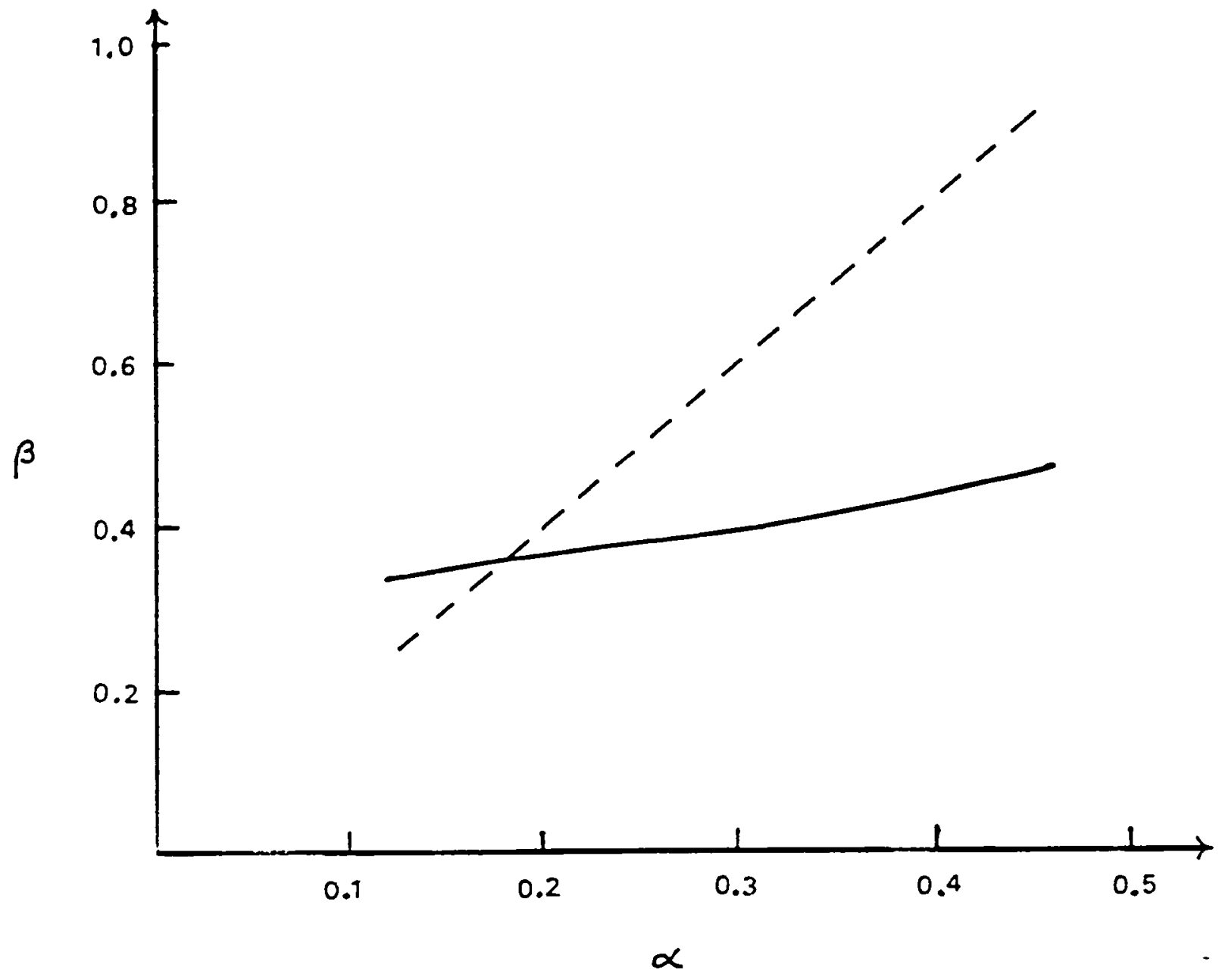

Figure 2. Values of the maximum value $\beta$ of $g(x)$ as a function of the initial value $g(0)=\alpha$. The dotted line is $\beta=2 \alpha$. 


$$
h
$$





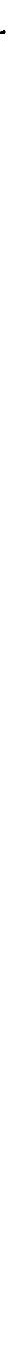




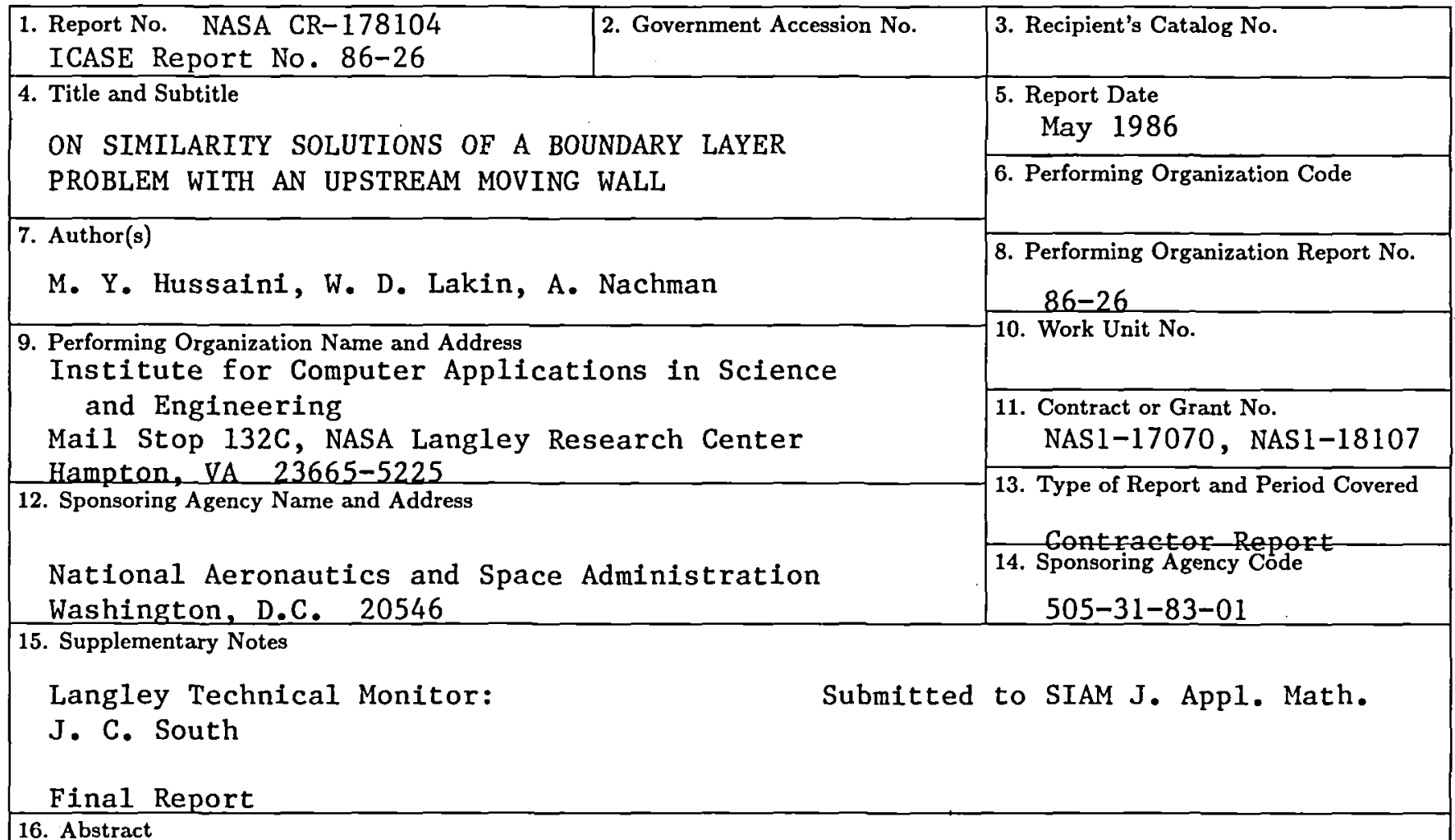

16. Abstract

This work deals with the problem of a boundary layer on a flat plate which has a constant velocity opposite in direction to that of the uniform mainstream. It has previously been shown that the solution of this boundary value problem is crucially dependent on the parameter which is the ratio of the velocity of the plate to the velocity of the free stream. In particular, it was proved that a solution exists only if this parameter does not exceed a certain critical value, and numerical evidence was adduced to show that this solution is nonunique. Using Crocco formulation the present work proves this nonuniqueness. A1so considered are the analyticity of solutions and the derivation of upper bounds on the critical value of wall velocity parameter.

\begin{tabular}{|c|c|c|c|c|}
\hline \multicolumn{2}{|c|}{ 17. Key Words (Suggested by Authors(s)) } & \multicolumn{3}{|c|}{ 18. Distribution Statement } \\
\hline \multicolumn{2}{|c|}{$\begin{array}{l}\text { existence, nonuniqueness, similarity, } \\
\text { solutions, Blasius equatior }\end{array}$} & \multicolumn{3}{|c|}{$\begin{array}{l}34 \text { - Fluid Mechanics and } \\
\text { Heat Transfer } \\
67 \text { - Theoretical Mathematics }\end{array}$} \\
\hline & & \multicolumn{3}{|c|}{ Unclassified - unlimited } \\
\hline $\begin{array}{l}\text { 19. Security Classif.( } \\
\text { Unclassifie }\end{array}$ & $\begin{array}{l}\text { (of this report) } \\
\text { d }\end{array}$ & $\begin{array}{l}\text { Classif.(of this page) } \\
\text { ified }\end{array}$ & $\begin{array}{l}\text { 21. No. of Pages } \\
25\end{array}$ & $\begin{array}{r}\text { 22. Price } \\
\mathrm{A} 022 \\
\end{array}$ \\
\hline
\end{tabular}




. 